\title{
THE ROLE OF THROMBIN IN CENTRAL NERVOUS SYSTEM ACTIVITY AND STROKE
}

\author{
ANCUȚA-MARIA PLEȘERU ${ }^{1,2}$, ROMEO GABRIEL MIHAILA $\breve{~}^{3}$
}

\author{
${ }^{1}$ Neurology Department, Faculty of Medicine, "Lucian Blaga" University of Sibiu, \\ Romania \\ ${ }^{2}$ Neurology Department, Emergency Clinical County Hospital Sibiu, Romania \\ ${ }^{3}$ Hematology Department, Faculty of Medicine, "Lucian Blaga" University of \\ Sibiu, Romania
}

\begin{abstract}
Background. Thrombin is a key factor of hemostasis, mediating the conversion of fibrinogen into fibrin. Along with prothrombin, of which thrombin is the active derivative, it has been found locally expressed in the central nervous system. This article aims to describe the role of thrombin in the normal functioning of the central nervous system and stroke.

Methods. In this mini-review, the specialized databases Medscape, PubMed, and Web of Science, from the years 2003-2018, were used to find relevant documents by using MeSH terms: "thrombin" and "stroke".

Results. Prothrombin and thrombin influence neural development, protection and regeneration, thrombin being a relatively strong regulating factor of brain function. However, high levels of thrombin are detrimental to neuronal health, and cause atherosclerotic plaque development and instability - a leading cause of cerebral infarction. In stroke, thrombin promotes direct cellular toxicity, vascular disruption, oxidative stress and inflammatory response. There is a direct correlation between thrombin activity in the affected brain hemisphere and the infarction volume. Direct acting thrombin inhibitors, like dabigatran, significantly decrease the risk of ischemic stroke.

Conclusion. Further studies on the correlation between thrombin levels, generation and activity and the risk and recurrence of ischemic cerebral stroke should give new insight on this association, resulting in an optimized practical therapeutic approach.
\end{abstract}

Keywords: central nervous system, thrombin, stroke

\section{Introduction}

Thrombin, as a major player in thrombogenesis, has been found to affect brain cells via receptor activation, both in normal development and function of the cerebrum, as well as in major cerebral diseases [1]. The role of thrombin generation levels and activity in acute ischemic stroke has been actively researched. Clear associations have been found between this coagulation factor and the severity of cerebral infarction [2]. On a more positive note, the proven neurovascular injury and high ischemic stroke risk caused by increased levels of thrombin led to it being considered as

Manuscript received: 25.01 .2018

Received in revised form: 03.04.2018

Accepted: 16.04.2018

Address for correspondence: ancutapleseru@yahoo.com a therapeutic target. Experimental studies have concluded that pretreatment with dabigatran, a direct thrombin inhibitor, alleviates the pro-inflammatory thrombin-induced tissue alteration and reduces blood-brain barrier leakage. The overall effect of dabigatran administration prior to ischemic stroke is the reduction in size of the infarcted aria, lack of increase in intracranial hypertension and a more favorable recovery of neurologic deficits [3]. Further data on the association between thrombin generation parameters, cardiovascular risk factors and cerebral infarction would lead to a more effective clinical and therapeutic approach of these pathological conditions.

Relevant scientific articles have been selected for this current minireview from several medical data bases, 
namely Medscape, PubMed, Web of Science, covering a time span of publication from 2003 up to 2018, and having as keyword: "thrombin" and "stroke".

The aim of this paper is to summarize and highlight the involvement of thrombin and it's activity in the main pathological processes involving the CNS, especially in acute and repetitive ischemic stroke. Thus, the importance of evaluating the generation, activity and levels of thrombin can be brought into focus for implementing future prevention and therapeutic solutions.

Thrombin involvement in physiological and pathological activity mechanisms of the central nervous system (CNS)

Thrombin is a coagulation factor belonging to the chymotrypsin serine proteases family. It has a central role in hemostasis, by mediating the conversion of fibrinogen into fibrin; thrombin is the activated derivative of prothrombin, being generated in the coagulation cascade by the combined actions of factors $\mathrm{V}$ and $\mathrm{X}$, in the presence of ionized calcium $(\mathrm{Ca} 2+)$ [4].

Recent studies have proved that prothrombin, along with thrombin, have been found locally in the central nervous system [5]. Notably, the central nervous system is the only site of thrombin expression, besides the liver [6]. In neurons and astrocytes, the expression of prothrombin has been proven by mRNA quantification.

Physiologically, prothrombin and thrombin influence neural development, protection and regeneration, thrombin being a relatively strong regulating factor of brain function [7].

The involvement of these two coagulation compounds in neurological diseases such as cerebral stroke, brain hemorrhage and trauma, epilepsy, Alzheimer's disease, multiple sclerosis and cerebral tumors is beginning to emerge [5].

In elevated quantities, thrombin is directly neurotoxic and promotes inflammation in endothelial cells, microglia and astrocytes. At least some of the cerebrovascular effects of hypoxia are mediated through thrombin [8]. In vitro, high levels of thrombin cause neuronal death, and in vivo, they contribute to blood-brain barrier alteration, brain edema and the initiation of seizures [9].

\section{Thrombin-mediated effects in acute ischemic stroke \\ Cerebral infarction, regardless of its etiology,} causes a central focal lesion of neuronal necrosis - the ischemic core, surrounded by an area of low blood perfusion - the ischemic penumbra. Thrombin is involved in stroke pathology via multiple pathways, including direct cellular toxicity, vascular disruption, oxidative stress and inflammatory response [10].

Increased thrombin activity and concentration are detrimental to neuronal health. Low levels of thrombin activity and concentration promote brain tissue viability, synaptic plasticity and have protective effects against excitotoxic detrimental factors [11].

During the acute phase of cerebral infarction, synaptic plasticity is altered through the activation of the excitatory N-Methyl-D-Aspartate receptors (NMDAR), which induces ischemic long-term potentiation of synaptic transmission. Experimental studies have proven that thrombin, by activating its own Protease-Activated receptor 1 (PAR1), mediates the same process of longterm potentiation of synaptic transmission. Furthermore, inhibition of thrombin or its receptor ceased the ischemic long-term potentiation of synaptic transmission and replaced it with the physiological functioning of interneuronal signaling [12].

Experimental studies in rats showed that thrombin increases the generation of mitochondrial and cytosolic reactive oxygen species, promotes nitric oxide generation and disrupts the tight junctions of the brain microvascular endothelial cells. Altogether, this mechanism underlines the blood-brain barrier dysfunction caused by thrombin [13].

The cumulus of this data proves that the high levels of thrombin activity and concentration during the acute stages of ischemic stroke contribute to the neurovascular injury. Thrombin generation via contact activation pathway is a proven risk factor for ischemic stroke [14].

\section{Thrombin as a valuable therapeutic target in preventing and treating cerebral infarction}

Given the large number of studies proving the involvement of thrombin generation and activity in the pathogenesis of ischemic stroke, this coagulation factor could potentially become a therapeutic target in preventing and treating cerebral infarction, and in reducing the magnitude of residual neurologic deficit following stroke.

Approximately one third of ischemic strokes are correlated with the atherosclerosis of the cervical portion of the carotid arteries. The pathway can be either embolization from parietal atherosclerotic lesions, or decreased blood flow due to local thrombosis or vascular stenosis [15]. Increased thrombin activity is correlated with atherosclerotic plaque development and instability. In turn, the increased local inflammatory activity further promotes thrombin generation, leading to pro-thrombotic alterations of the blood and of the carotid wall [16].

Atrial fibrillation is one of the most important risk factors for ischemic stroke. In such cases, anticoagulation mainly by using coumarin medication, is a common way of reducing cerebral infarction [17]. Thrombin inhibitors with direct action, such as dabigatran etexilate, an orally prescribed anticoagulant, significantly decrease the risk of ischemic stroke in patients with atrial fibrillation, as opposed to less efficient formerly used anticoagulants. It also reduces the risk of major bleeding [18]. Two other newly developed, available and orally prescribed anticoagulants 
are rivaroxaban and apixaban which act as factor $\mathrm{X}$ inhibitors. Although the new oral anticoagulants (NOACs) have a safer profile than previous similar drugs when it comes to bleeding risk, there are two main challenges faced while prescribing them. Firstly, the association of NOACs with amiodarone, an antiarrhythmic drug widely prescribed for patients with atrial fibrillation, significantly increases the risk of bleeding in these patients. Secondly, out of the three NOACs, dabigatran, the direct inhibitor of thrombin, is the only one which has a specific antidote in case of overdosing, the monoclonal antibody idarucizumab. The availability of this antidote is currently limited due to it being expensive to manufacture and purchase [19].

Recombinant tissue plasminogen activator (rTPA) is the first choice in treating selected patients affected by ischemic stroke. It has been proven to induce a notable reduction of thrombin generation after 24 hours following the initiation of the treatment [20].

To reduce the risk of recurrent stroke, acetylsalycilic acid is the most commonly used platelet antiaggregant. It is given to patients with embolic cerebral infarction of undetermined source. Clinical trials are being conducted to assess whether the oral inhibitor of thrombin, dabigatran etexilate, is more efficient than acetylsalycilic acid in preventing future ischemic strokes [21].

\section{Thrombin involvement in hemorrhagic stroke}

Thrombin also plays an emerging role in the physiopathology of hemorrhagic stroke. High levels of thrombin were found to take part in the development of brain edema in intracerebral hemorrhage [22]. Furthermore, thrombin is involved in seizure activity in hemorrhagic stroke, by increasing neuronal excitability as soon as it enters the brain tissue. This is particularly prominent in the immature brain of newborns. In these cases, thrombin alters the resting membrane potential by amplifying the current through voltage-gated sodium channels [11].

\section{Conclusion}

By reviewing the physiological implications of thrombin within central nervous system (CNS), this coagulation factor proves to be a newly emerging key factor in brain function, regulation and inter-neuronal signaling. Pathologically, it is involved in prominent cerebral diseases such as ischemic stroke, brain trauma, seizure activity and blood-brain barrier dysfunction. The main positive outcome of these findings is the possibility of targeting thrombin for preventing, curing and limiting the impact of cerebrovascular diseases on general health. This would be important concerning the population groups with risk factors such as atrial fibrillation, hypertension, carotid atherosclerosis, thrombophilia, diabetes, hyperlipidemia and procoagulant states. In this matter, further research on the correlation between thrombin levels, generation and activity and the risk and recurrence of ischemic cerebral stroke is fundamental. The result would be an optimized, practical prophylactic and therapeutic approach in patients with ischemic stroke.

\section{References}

1. Bushi D, Ben Shimon M, Shavit Stein E, Chapman J, Maggio N, Tanne D. Increased thrombin activity following reperfusion after ischemic stroke alters synaptic transmission in the hippocampus. J Neurochem. 2015;135(6):1140-1148.

2. Bushi D, Chapman J, Katzav A, Shavit-Stein E, Molshatzki N, Maggio N, Tanne D. Quantitative detection of thrombin activity in an ischemic stroke model. J Mol Neurosci. 2013;51(3):844-850.

3. Dittmeier M, Wassmuth K, Schuhmann MK, Kraft P, Kleinschnitz C, Fluri F. Dabigatran Etexilate Reduces ThrombinInduced Inflammation and Thrombus Formation in Experimental Ischemic Stroke. Curr Neurovasc Res. 2016;13(3):199-206.

4. Loeffen R, Winckers K, Ford I, Jukema JW, Robertson M, Stott DJ, et al. Associations Between Thrombin Generation and the Risk of Cardiovascular Disease in Elderly Patients: Results From the PROSPER Study. J Gerontol A Biol Sci Med Sci. 2015;70(8):982-988.

5. Krenzlin H, Lorenz V, Danckwardt S, Kempski O, Alessandri B. The Importance of Thrombin in Cerebral Injury and Disease. Int J Mol Sci. 2016 Jan 11;17(1). doi: 10.3390/ijms17010084.

6. Krenzlin H, Lorenz V, Alessandri B. The involvement of thrombin in the pathogenesis of glioblastoma. J Neurosci Res. 2017;95(10):2080-2085.

7. Maragoni MN, Braun D, Situ A, Moyano AL, Kalinin S, Polak $\mathrm{P}$, et al. Differential effects on glial activation by a direct versus an indirect thrombin inhibitor. J Neuroimmunol. 2016;297:159-168. 8. Grammas P, Martinez JM. Targeting thrombin: an inflammatory neurotoxin in Alzheimer's disease. J Alzheimers Dis. 2014;42 Suppl 4:S537-S544.

9. Xi G, Reiser G, Keep RF. The role of thrombin and thrombin receptors in ischemic, hemorrhagic and traumatic brain injury: deletrious or protective? J Neurochem. 2003;84(1):3-9.

10. Chen B. Thrombin in Ischemic Stroke Targeting. In Lapchak PA, Zhang JH (eds). Translational Stroke Research: From Target Selection to Clinical Trials; 2012, pp 189-204.

11. Garcia PS, Ciavatta VT, Fidler JA, Woodbury A, Levy JH, Tyor WR. Concentration-Dependent Dual Role of Thrombin in Protection of Cultured Rat Cortical Neurons. Neurochem Res. 2015;40(11):2220-2229.

12. Stein ES, Itsekson-Hayosh Z, Aronovich A, Reisner Y, Bushi D, Pick CG, et al. Thrombin induces ischemic LTP (iLTP): implications for synaptic plasticity in the acute phase of ischemic stroke. Sci Rep. 2015 Jan 21;5:7912.

13. Brailoiu E, Shipsky MM, Yan G, Abood ME, Brailoiu GC. Mechanisms of modulation of brain microvascular endothelial cells function by thrombin. Brain Res. 2017;1657:167-75.

14. Olson NC, Butenas S, Lange LA, Lange EM, Cushman M, Jenny NS, et al. Coagulation factor XII genetic variation, ex vivo thrombin generation, and stroke risk in the elderly: results from the Cardiovascular Health Study. J Thromb Haemost. 2015;13(10);1867-1877.

15. Qaja E, Bhimji S, Carotid Artery Stenosis, StatPearls Publishing, 2017. Available from: https://www.ncbi.nlm.nih.gov/ books/NBK442025/

16. Kalz J, ten Cate H, Spronk HM. Thrombin generation and atherosclerosis. J Thromb Thrombolysis. 2014;37(1):45-55.

17. Winstanley L, Chen R. New thrombin and factor Xa inhibitors 
for primary and secondary prevention of ischaemic stroke. CNS Neurol Disord Drug Targets. 2013;12(2):242-251.

18. Serebruany V, Sani Y, Lynch D, Schevchuck A, Svetlov S, Fong A, et al. Effects of dabigatran in vitro on thrombin biomarkers by Calibrated Automated Thrombography in patients after ischemic stroke. J Thromb Thrombolysis. 2012;33(1):22-27.

19. Douglas S, Paauw MD. NOACs for Atrial Fibrillation: Important Drug/Drug Interactions. Medscape - Jan 03, 2018. Available from: https://www.medscape.com/viewarticle/890656. 20. Goldman S, Prior SM, Bembenek JP, Niewada M, Broniatowska E, Czlonkowska A, et al. Activation of blood coagulation and thrombin generation in acute ischemic stroke treated with rtPA. J Thromb Thrombolysis. 2017;44(3):362-370. 21. Diener HC, Easton JD, Granger GB, Cronin L, Duffy C, Cotton D, et al. Design of Randomized, double-blind, Evaluation in secondary Stroke Prevention comparing the EfficaCy and safety of the oral Thrombin inhibitor dabigatran etexilate vs. acetylsalicylic acid in patients with Embolic Stroke of Undetermined Source (RE-SPECT ESUS). Intl J Stroke. 2015;10(8):1309-1312.

22. Isaeva E, Hernan A, Isaev D, Holmes GL. Thrombin facilitates seizures through activation of persistent sodium current. Ann Neurol. 2012;72(2):192-198. 